\title{
Skin-, nipple-, and areola-sparing mastectomy
}

\author{
E. M. Umoh, N. Arora, R. M. Simmons \\ Department of Surgery, New York Presbyterian Hospital, The Weill Medical College of Cornell University, \\ New York, NY, USA.
}

\begin{abstract}
Surgical management of breast carcinoma has evolved to include more breast conserving techniques such as skin-, nipple-, and areola-sparing mastectomies, as improved cosmesis becomes an increasing concern. However, the oncologic risk of these procedures must be strongly considered before such techniques can be widely adopted. Here we review available literature on these techniques and their associated clinical outcome. From our own experience, as well as from that reported, we conclude that nipple-, skin-, and areola-sparing mastectomies in carefully selected patients can have safe oncologic outcomes comparable to more traditional surgical techniques and therefore may be a feasible option for breast cancer management.
\end{abstract}

Keywords: Areola preservation; Breast reconstruction; Nipple-sparing mastectomy; Skin-sparing mastectomy

\section{Introduction}

Historically, breast cancer was managed by radical mastectomy. Since then, the pendulum has turned such that the majority of breast cancer is safely managed with breast conserving therapy. However, some women still require or prefer mastectomy and a number of advances in surgical technique including both oncologic resection and reconstruction have allowed for improved esthetic outcome.

Skin-sparing mastectomy (SSM), nipple-sparing mastectomy (NSM), and areola-sparing mastectomy (ASM) have all been described in recent literature and have been tested for oncologic safety and patient satisfaction with esthetic outcome. These techniques all provide improved cosmesis, especially when coupled with immediate breast reconstruction (IBR).

Correspondence to: Rache M. Simmons, MD, Weill Cornell Breast Center, 425 East 61st Street, 8th Floor, New York, NY 20021, USA. E-mail: rms2002@med.cornell.edu; Tel: 212-821-0853; Fax: 212-821-0832

Received: $21 / 10 / 08$

Accepted: 01/12/08

BCO/768/2008/FO
The following is a brief description and literature review of SSM, NSM, and ASM for the management of breast carcinoma.

\section{Skin-sparing mastectomy}

\section{Surgical technique and considerations}

SSM includes removal of all breast parenchyma, any existing biopsy scar, and complete resection of the nipple/areolar complex (NAC). The surgical oncologist and reconstructive surgeon work together to select the most desirable incision - three of the most common being periareolar, modified elliptical, and mastopexy/reduction mastoplasty [1]. Modifications such as axillary incisions or mediallateral extensions can be added to increase exposure to axillary contents, prior biopsy scars, and tumor areas [2]. Sentinel node biopsy and/or axillary dissection can also be performed when indicated for invasive disease.

Options for breast reconstruction for SSM include tissue expander/implant, transverse rectus abdominis myocutaneous flap (TRAM), deep inferior epigastric perforator (DIEP) flap, latissimus dorsi 
Table 1. Literature review of oncologic outcomes of skin-sparing mastectomy with immediate breast reconstruction.

\begin{tabular}{|c|c|c|c|c|c|c|}
\hline Author & $n$ & $\begin{array}{l}\text { Mean F/U } \\
\text { (months) }\end{array}$ & $\begin{array}{l}\text { Stage }(0 \text { or I) } \\
(\%)\end{array}$ & LR (\%) & DR (\%) & Notes \\
\hline Simmons [2] & 77 & 15.6 & 46.8 & 3.9 & 3.9 & $\begin{array}{l}\text { No recurrence in patients with } \\
\text { stage } 0-\text { Il disease }\end{array}$ \\
\hline Omranipour [4] & 95 & 69 & 74.6 & 1.1 & 2.1 & $\begin{array}{l}\text { LR in axilla, DR in bone and liver, } \\
\text { one patient with both LR and DR }\end{array}$ \\
\hline Salhab [5] & 21 & 13.5 & NR & 0 & 5 & - \\
\hline Foster [6] & 67 & 49.2 & 55 & 4 & 16 & All LR, DR in stage $\geqslant I I I A$ \\
\hline Carlson [9] & 539 & 65.4 & 54.9 & 5.5 & NR & $\begin{array}{l}\text { Tumor stage, high grade, } \\
\text { lymphovascular invasion } \\
\text { associated with recurrence }\end{array}$ \\
\hline Spiegel [10] & 221 & $\begin{array}{l}117 \text { - invasive } \\
120 \text { - DCIS }\end{array}$ & NR & 5.6 (invasive) & 6.8 (invasive) & $\begin{array}{l}\text { No evidence of recurrence in } \\
\text { DCIS (all subtypes) }\end{array}$ \\
\hline
\end{tabular}

F/U: follow-up; LR: local recurrence; DR: distant recurrence; DCIS: ductal carcinoma in situ; NR: not recorded.

flap, or gluteal flap [1]. Selection depends on individual patient characteristics. Complications of SSM with IBR include flap necrosis, native skin necrosis, hematoma, and implant failure [1]. The incidence of flap and skin necrosis varies between $0 \%$ and $12 \%$ across studies [4-7]; however, overall complication rates were not found to be significantly different compared to non-SSM (NSSM). One report by Carlson and colleagues showed that native skin flap epidermolysis occurred in $10.7 \%$ of patients with SSM and in $11.2 \%$ of those with NSSM ( $P=$ non-significant) [7].

\section{Oncologic safety}

A number of both retrospective and prospective studies over the past decade have verified the oncologic safety of SSM in select patients. Our group retrospectively investigated local recurrence (LR) and distant recurrence (DR) in patients receiving SSM $(n=77)$ and NSSM $(n=154)$ [2]. After a mean follow-up of 15.6 months, both LR and DR rates were not significantly different between the two groups, along with 5-year actuarial disease-free survival for LR and DR. Likewise, the 5-year overall survivals between SSM and NSSM groups were similar at $7.8 \%$ and $6.5 \%$, respectively. A similar retrospective study by Rivadeneira and colleagues with a different group and having a mean follow-up time of 38.4 months reported similar findings [8].

Outcomes data of several recent studies of SSM are listed in Table 1. LR varied between $0 \%$ and $5.6 \%$, while DR varied between $2.1 \%$ and $16 \%$ $[2,4-6,9,10]$. An important consideration in the variability demonstrated in recurrence rates, particularly DR, is the differences in the distribution of disease stage. Foster et al. investigated outcomes in both early- and late-stage disease. They found DR as high as 16\%; however, all these occurred in patients with stage IIIA disease or higher [7]. In all but one of the studies listed, there were no recurrences in patients with stage 0 or stage I disease, and in the remaining study, only six out of 539 patients (5.5\%) with early disease had LR [9]. Similarly, Downes et al. investigated the utility of SSM with IBR in 38 patients with high-risk breast cancer (disease stage IIA or greater; mean followup: 52.9 months). LR was seen in $2.6 \%$, while DR was seen in $26.3 \%$ [11].

In addition to disease stage, other tumor factors related to disease recurrence after SSM include poor tumor differentiation, lymphovascular invasion, tumor size greater than $2 \mathrm{~cm}$, and node-positive disease $[2,9,10]$. These data indicate that careful patient selection is necessary to minimize cancer recurrence in patients receiving SSM.

\section{Nipple-sparing mastectomy}

\section{Surgical technique and considerations}

Similar to SSM, NSM involves removal of all breast parenchyma and previous scars; however, the skin envelope, including the dermis and epidermis of the nipple and areola, is left intact. Importantly, the nipple core should be sent for frozen section and any evidence of NAC involvement precludes the procedure, as removal of the nipple remnant is necessary. Similarly, the final pathology positive for NAC involvement necessitates further management either with repeat surgery and NAC removal or with other therapy, such as radiation. Sentinel node biopsy and axillary dissections can be performed as indicated.

Careful planning of the incision is critical, as consideration must be given not only to prior biopsy scars, tumor location, and reconstructions but also 
to NAC vascularization. Sacchini described four different skin incisions for NSM, including an inferior or superior periareolar incision with lateral extension, which offers good surgical exposure but may compromise blood supply to the flap periphery and areola; transareolar with perinipple and lateralmedial extension, with the possible sequelae of perinipple artery division or scarring; transareolar and transnipple with extensions; or a mammary crease incision inferiorly or laterally, which may offer the best preservation of flap vascularity [13]. Radial transareolar incisions have been associated with reduced NAC loss and improved surgical outcomes $[14,15]$.

The most important technical concern is the preservation of adequate vascularization to the NAC to prevent necrosis. The vascular anatomy of the NAC shows significant variation in sources and patterns of blood supply between individuals. The internal and lateral thoracic, internal mammary, and intercostal arteries have been shown to contribute in varying and overlapping amounts to NAC vascularization. Both radial and loop anastomotic vascular patterns have been described, and these patterns have important surgical implications in the maintenance of sufficient NAC blood supply. One study reported NAC necrosis as high as $11 \%$, almost half of which was in the setting of TRAM reconstruction. As in SSM, other possible complications include flap or skin necrosis, wound infection, and implant loss [13].

Considerations for the type of reconstruction slightly differ for NSM than for SSM. Importantly, tension on the skin via subcutaneous implants may impact blood supply to the skin and NAC. Tissue expanders and muscle flaps such as latissumus dorsi, TRAM, and DIEP have been utilized, although there appears to be lower complication rates with initial tissue expander use $[13,14]$. Sacchini et al. [13] investigated 192 NSMs in 123 patients receiving either subpectoral or subcutaneous implants, tissue expanders, or TRAM reconstructions. Complications included flap necrosis, nipple/areola necrosis, or implant loss when applicable. Complications occurred in $22 \%$ of those receiving subpectoral or subcutaneous reconstruction, in $13 \%$ of those receiving tissue expanders, and in $32 \%$ of TRAM reconstructions.

\section{Oncologic safety}

Although offering improved cosmesis, the oncologic consequence of NSM must be thoroughly considered, as any occult cancer or remaining breast tissue poses a significant risk to the patient. Reported rates of malignancy involvement within the nipple range from $0 \%$ to $58 \%$, depending on the size of the primary breast tumor, location, multicentricity, and lymph node positivity [13,16-20]. Our group retrospectively investigated 217 mastectomy specimens for malignant involvement of the nipple, areola, or both. We found $10.6 \%$ of specimens had nipple involvement. However, when a subgroup of patients with tumors $<2 \mathrm{~cm}$, peripheral tumors, and two or fewer positives nodes was analyzed, the rate dropped to $6.7 \%$ [17]. In another study, factors found to be associated with NAC preservation were tumor nipple distance $>4 \mathrm{~cm}$ and absence of lymphovascular invasion [21].

Table 2 summarizes cancer outcomes in recent studies of NSM with IBR. LR ranged from $2 \%$ to $24 \%$, while DR ranged from $1.4 \%$ to $20 \%$. Overall patient survival ranged from $76.4 \%$ to $100 \%$ [13,22-24]. Benediktsson and Perbeck [23] reported much higher LR and DR rates, and the lower overall survival compared to other studies was due to subcutaneous mastectomy being used, which the authors attributed to comparatively reduced rates of adjuvant radiation use. Importantly, in all the studies listed, patients with small tumor size, early-stage disease, and peripherally located tumors were preferentially selected, indicating that NSM may be more beneficial in this select subgroup of patients; however, a risk of recurrence is not negligible and patients must be counseled and followed appropriately.

Table 2. Literature review of oncologic outcomes of nipple-sparing mastectomy with immediate breast reconstruction for breast cancer.

\begin{tabular}{|c|c|c|c|c|c|c|}
\hline Author & $n$ & Mean F/U (months) & LR (\%) & DR (\%) & OS (\%) & Notes \\
\hline Sacchini [13] & 68 & 24.6 & 3 & 1.4 & 98.6 & $\begin{array}{l}\text { LR in UOQ; time to LR } 71.8 \text { and } 6.0 \\
\text { months; } 1 \text { fatality with DR }\end{array}$ \\
\hline Caruso [22] & 50 & 66 & 2 & 10 & 92 & LR in nipple; four fatalities with DR \\
\hline Benediktsson [23] & 216 & 156 & 24 & 20 & 76.4 & $\begin{array}{l}\text { Reduced adjuvant radiation in patients; } \\
\text { LR } 8.5 \% \text { in irradiated patients }\end{array}$ \\
\hline Petit (IORT) [24] & 570 & 19 & 2.4 & 2.3 & 100 & LRs near primary tumor, none in NAC. \\
\hline
\end{tabular}

F/U: follow-up; LR: local recurrence, DR: distant recurrence, OS: overall survival, NAC: nipple-areolar complex, UOQ: upper outer quadrant; IORT: intraoperative radiation therapy. 


\section{Areola-sparing mastectomy}

\section{Surgical technique and considerations}

The ASM technique includes resection of the nipple, any existing biopsy scar, and removal of all breast parenchyma; however, the areola is preserved. A minimal amount of skin is removed, similar to SSM, which provides a natural envelope of skin for superior reconstruction. Incisions may be completely intra-areolar (either S-shaped or linear), linear intra-areolar with extra-areolar extension, inverted-T infra-mammary crease, or infra-mammary crease and perinipple, with or without additional axillary incision. The undersurface of the areolar flap can be touch-prepped for cytological evaluation at the discretion of the surgical oncologist if there is concern regarding margins in this area. Complications and reconstruction options are similar to those for SSM.

\section{Oncologic safety}

Sparing the areola improved reconstruction with either prosthetic implants or flaps. This patient satisfaction is justified, however, only if oncologic safety is not compromised. While numerous studies have investigated the malignant involvement of the NAC in breast cancer, the anatomic differences between the nipple and areola, however, necessitate separate investigation of possible malignant involvement $[25,26]$. In our pathologic study of 217 mastectomy specimens, we found malignant areola involvement to be less than $1 \%$ for patients with small, infiltrating carcinoma and no areola involvement in DCIS [17]. With these data, we retrospectively analyzed 17 ASMs in 12 patients with IBR. Mean patient follow-up was 7 months (range 2-17 months). Ten ASMs were performed for breast cancer prophylaxis, four for DCIS, and three for infiltrating ductal carcinoma. Thirteen were done with tissue expanders and the remaining with TRAM flaps. There was one post-operative complication (local wound infection) and no instance of LR or DR [27].

Our data indicate that ASM may be a safe option for selected patients; however, prospective trials that are currently ongoing will provide valuable additional information on the safety and efficacy of this technique.

\section{Conclusion}

With meticulous patient selection and well-planned surgical technique, conservation of the skin, areola, and possibly the nipple can provide satisfactory oncologic and esthetic outcomes with minimal complications and improved patient satisfaction.

\section{References}

1. Simmons RM. Skin-sparing mastectomies. Am J Surg 2000; 180: 290-293.

2. Simmons RM, Fish SK, Gayle L, et al. Local and distant recurrence rates in skin-sparing mastectomies compared with non-skin-sparing mastectomies. Ann Surg Oncol 1999; 6: 676-681.

3. Allweis TM, Boisvert ME, Otero SE, et al. Immediate reconstruction after mastectomy for breast cancer does not prolong the time to starting adjuvant chemotherapy. Am J Surg 2002; 183: 218-223.

4. Omranipour R, Bobin J, Esouyeh M. Skin sparing mastectomy and immediate breast reconstruction (SSMIR) for early breast cancer: Eight years single institution experience. World J Surg Oncol 2008; 6: 43.

5. Salhab M, Sarakb WA, Joseph A, et al. Skin-sparing mastectomy and immediate breast reconstruction: patient satisfaction and clinical outcome. Int $J$ Clin Oncol 2006; 11: 51-54.

6. Foster RD, Esserman LJ, Anthony JP, et al. Skin-sparing mastectomy and immediate breast reconstruction: a prospective cohort study for the treatment of advanced stages of breast carcinoma. Ann Surg Oncol 2002; 9: 462-466.

7. Carlson GW, Bostwick J, Styblo TM, et al. Skin-sparing mastectomy: oncologic and reconstructive considerations. Ann Surg 1997; 225: 570-578.

8. Rivadeneira DE, Simmons RM, Fish SK, et al. Skinsparing mastectomy with immediate breast reconstruction: a critical analysis of local recurrence. Cancer $J$ 2000; 6: 331-335.

9. Carlson GW, Styblo TM, Lyles RH. Local recurrence after skin-sparing mastectomy: tumor biology or surgical conservatism? Ann Surg Oncol 2002; 10: 108-112.

10. Spiegel AJ, Butler CE. Recurrence following treatment of ductal carcinoma in situ with skin sparing and immediate breast reconstruction. Plast Reconstr Surg 2003; 111: 706-711.

11. Downes KJ, Glatt BS, Kanchwala, et al. Skin-sparing mastectomy and immediate reconstruction is an acceptable treatment option for patients with high-risk breast carcinoma. Cancer 2005; 103: 906-913.

12. Medina-Franco $\mathrm{H}$, Vasconez $\mathrm{O}$, Fix $\mathrm{J}$, et al. Factors associated with local recurrence after skin-sparing mastectomy and immediate breast reconstruction for invasive breast cancer. Ann Surg 2002; 235: 814-819.

13. Sacchini V, Pinotti JA, Barros A, et al. Nipple-sparing mastectomy for breast cancer and risk reduction: oncologic or technical problem. J Am Coll Surg 2006; 203: 704-714.

14. Wijayanayagam A, Kumar AS, Roster RD. Optimizing the total skin-sparing mastectomy. Arch Surg 2008; 143: 38-45.

15. Stolier AJ, Sullivan SK, Dellacroce FJ. Technical considerations in nipple-sparing mastectomy: 82 consecutive cases without necrosis. Ann Surg Oncol 2008; 15: 1341-1347.

16. Laronga C, Kemp B, Johnston D, et al. The incidence of occult nipple-areola complex involvement in breast cancer patients receiving a skin-sparing mastectomy. Ann Surg Oncol 1999; 6: 609-613. 
17. Simmons RM, Brennan M, Christos $P$, et al. Analysis of nipple/areolar involvement with mastectomy: Can the areola be preserved? Ann Surg Oncol 2002; 9: 165-166.

18. Menon RS, van Geel AN. Cancer of the breast with nipple involvement. Br J Cancer 1989; 59: 81-84.

19. Smith J, Payne WS, Carney JA. Involvement of the nipple and areola in carcinoma of the breast. Surg Gynecol Obstet 1976; 143: 546-548.

20. Verma GR, Kumar A, Joshi K. Nipple involvement in peripheral breast carcinoma: a prospective study. Indian J Cancer 1997; 34: 1-5.

21. Vlajcic Z, Rado Z, Standec S, et al. Nipple-areola complex preservation: predictive factors of neoplastic nipple-areola complex invasion. Ann Plast Surg 2005; 55: 240-244.

22. Caruso F, Ferrara M, Castiglione G, et al. Nipple sparing subcutaneous mastectomy: 66 months follow-up. EJSO 2006; 32: 937-940.
23. Benediktsson KP, Perbeck L. Survival in breast cancer after nipple-sparing subcutaneous mastectomy and immediate reconstruction with implants: A prospective trial with 13 years median follow-up in 216 patients. EJSO 2008; 34: 143-148.

24. Petit JY, Veronesi U, Orecchia R, et al. Nipple-sparing mastectomy in association with intra operative radiotherapy (ELIOT): a new type of mastectomy for breast cancer management. Breast Cancer Res Treat 2006; 96: 47-71.

25. Giacometti L, Montagna W. The nipple and the areola of the human female breast. Anat Rec 1992; 144: 191-197.

26. Schnitt SF, Goldwyn RM, Slavin SA. Mammary ducts in the areola: implications for patients undergoing reconstructive surgery of the breast. Plast Reconstr Surg 1993; 92: 1290-1293.

27. Simmons RM, Hollenbeck ST, Latrenta GS. Areola-sparing mastectomy with immediate breast reconstruction. Ann Plast Surg 2003; 51: 547-551. 